\title{
Mature rapid response system and potentially avoidable cardiopulmonary arrests in hospital
}

\section{Sanjay Galhotra, Michael A DeVita, Richard L Simmons, Mary Amanda Dew; and members of the Medical Emergency Response Improvement Team (MERIT) Committee}

See end of article for authors' affiliations

Correspondence to: Dr Michael A DeVita University of Pittsburgh Medical Center, Presbyterian Hospital, 200 Lothrop Street, Pittsburgh, Pennsylvania 15213,USA; devitam@ upmc.edu

Accepted 30 April 2007

\begin{abstract}
Objective: To study the incidence, outcome and potentially avoidable causes of inpatient cardiopulmonary arrests in a hospital with a "mature" rapid response system (RRS).

Design: Retrospective observational study of all cardiopulmonary arrest events in 2005.

Setting: University of Pittsburgh Medical Center Presbyterian Hospital, a 730-bed academic, urban, tertiary care adult hospital in the USA.

Interventions: None.

Results: During the calendar year 2005, the 16th year since the establishment of a medical emergency team (MET)/RRS, the MET was activated 1942 times; 111 of these events were cardiopulmonary arrest events (3.26 arrest events/1000 patient admissions), and 1831 were non-arrest patient crisis events (53.8 crisis events/1000 patient admissions). A review of the 104 index cardiopulmonary arrest events revealed that 26 $(25 \%)$ patients survived to discharge. Event survival decreased as the intensity of patient monitoring decreased (83\% in intensive care units, $69 \%$ in monitored, and $36 \%$ in unmonitored units; $p=0.002$ ), but the rate of subsequent inhospital death was higher in the more intensely monitored settings $160 \%, 38 \%, 23 \%$, respectively; $p=0.022$ ). Nineteen $(18 \%)$ arrests were deemed to be "potentially avoidable". Avoidable arrests were classified as: failure to adhere to established hospital patient care guideline or policy; inadequate monitoring or surveillance; or delays in dealing with patient needs including delay in MET/RRS activation.

Conclusions: In spite of the high crisis event rate and a low rate of cardiac arrests, potentially avoidable cardiopulmonary arrests still occurred. According to the present study more cardiopulmonary arrest events might be avoided by better adherence to hospital patient care policies, by closer monitoring on floors and by preventing delays in addressing deterioration in patient condition.
\end{abstract}

$\mathrm{S}$ uccessful resuscitation and survival to discharge after a cardiopulmonary arrest have been linked to several factors-witnessing of arrest, early initiation of resuscitation, return of cardiac function within $20 \mathrm{~min}$, young age, ${ }^{1}$ patient monitoring ${ }^{2}$ and time of day. ${ }^{3}$ Nevertheless, rates of survival to discharge after an adult inhospital cardiopulmonary arrest remain poor, ${ }^{4-6}$ and, prevention of cardiopulmonary arrests remains the best strategy to decrease inhospital patient mortality.

Medical emergency team (MET)/rapid response systems (RRS) have been proposed as a strategy to better anticipate and thus prevent inhospital cardiopulmonary arrests. ${ }^{7-9}$ Since up to $80 \%$ of cardiopulmonary arrest events are preceded by prolonged periods of physiological and clinical instability, ${ }^{10-13}$ intervening early during this period in the form of a MET/RRS crisis call should, at least in theory, help in preventing some cardiopulmonary arrests. In practice, there is evidence for ${ }^{14-16}$ and against this hypothesis. ${ }^{17}$ More research is needed to further understand the role of a MET/RRS in the prevention of cardiopulmonary arrests. Recent reports have stated that facilitating additional MET/RRS crisis calls might help in avoiding some cardiopulmonary arrests. ${ }^{18} 19$ But inefficient crisis detection ${ }^{19}{ }^{20}$ and delays in activating a MET/RRS response ${ }^{13}$ have been cited as problems that could minimise the benefit of a MET/RRS intervention.

Several questions still remain unanswered. It is not known whether, and to what extent, a well-established and "mature" RRS (having all four components: an "afferent" component (crisis detection), an "efferent" component (the crisis response teams, physician or nurse led), an "evaluative or process improvement" component, and an "administrative" component) can eliminate "potentially avoidable" cardiopulmonary arrests in an inpatient setting. ${ }^{9}$ If avoidable cardiopulmonary arrests continue to occur despite widespread use of the MET/ RRS, then what are the patient and event characteristics of those events, and more importantly, what strategies can be adopted to eliminate the avoidable events? With respect to the latter, are more MET/RRS calls needed or are other strategies required?

This investigation aimed to study the incidence, outcome and potentially avoidable causes of inpatient cardiopulmonary arrests in a hospital with a "mature" RRS.

\section{METHODS}

This project was approved by the Quality Improvement Review Committee, in accordance with the Patient Safety Committee and the Total Quality Council. Our organisation requires approval by these entities for quality improvement projects.

\section{Setting}

The University of Pittsburgh Medical Center Presbyterian Hospital Pittsburgh, Pennsylvania, USA, is an adult, 730-bed tertiary care hospital. It has 160 intensive care beds, 330 monitored beds and 240 unmonitored beds. The RRS was established in 1989 but use remained low for many years due to cultural barriers in the workplace and lack of standardisation. After objective activation criteria were outlined and implemented

Abbreviations: CCM, critical care medicine; ICU, intensive care unit; MET, medical emergency team; RRS, rapid response system 
hospital-wide in December 2000, MET activation markedly increased. ${ }^{16} 21$

The MET response is led by a critical care medicine (CCM) faculty member, and the rest of the team is composed of a CCM fellow, two intensive care unit (ICU) nurses, and two respiratory therapists. ${ }^{22}$ It can be activated by anyone (staff, patient or family) and anywhere in the hospital, $24 \mathrm{~h}$ a day and 7 days a week. ${ }^{20}$

\section{Data collection and event analysis}

We used the hospital's code database to identify all inpatient cardiopulmonary arrests during the year 2005. The code database is a log of all cardiopulmonary arrests (referred to as condition A in the hospital) and crisis calls (referred to as condition C). ${ }^{20}$ Each time a condition $\mathrm{A}$ or $\mathrm{C}$ call is made, the telephone operator records the following event variables: date, time, location, call type and patient identifiers. This information is then entered into an electronic database. The Hospital Code Review Committee (comprising the medical director, a senior CCM faculty member, a CCM fellow, a patient safety fellow, and a nursing unit director) reviews all cardiopulmonary arrest events, and a convenience sample (about $40 \%$ ) of crisis events. For the present project, a physician (SG) reviewed the medical records of all patients suspected to have had a cardiopulmonary arrest to extract relevant information, and presented each event to the review committee. Discussions were focused on the clinical events which preceded the event, whether the arrest was a true cardiopulmonary arrest, whether the arrest was "predictable", whether the arrest was "potentially avoidable", and if there were possible prevention strategies. Decisions about "predictability" and "potential avoidability" (defined below) were based on consensus among one nurse and four physician members of the code review team. For patients experiencing multiple arrests during their hospital stay, only the first cardiopulmonary arrest event and resuscitation were considered for analysis. Neurological outcome was measured by applying the Cerebral Performance Category scale to the extracted medical record data. ${ }^{23}$

\section{Definitions}

\section{Cardiopulmonary arrest}

An event was determined to be a true cardiopulmonary arrest if monitoring data showed presence of a non-perfusing rhythm (eg, asystole or ventricular fibrillation) or the responding team had documented that the patient was unresponsive, pulseless and apnoeic.

\section{Monitoring}

At our institution and elsewhere, patient monitoring is defined by both the level of technology used and the personnel who must therefore be present to maintain that level of surveillance. In terms of technology, we considered a patient to be "monitored" when continuous pulse oximetry or continuous ECG monitoring or both were being used at the time of arrest event. In terms of surveillance by hospital staff, an unmonitored unit usually has a nurse to patient ratio of $1: 6$ and a monitored unit has a nurse to patient ratio of 1:4. All ICU patients are multimodality monitored (eg, pulse oximetry, ECG, blood pressure), nurse to patient ratio is $1: 2$, and a boardcertified intensivist is in the hospital at all times.

\section{Predictability}

Events were termed "predictable" if the patient chart indicated objective or clear evidence of patient deterioration in the 6-h period before the arrest event.
Potential avoidability

Events were termed "potentially avoidable" if one or more of the following was noted in the time leading up to the arrest event and, if avoided, might have either prevented the arrest or changed the arrest outcome. The code review team determined this during the detailed review of the event.

- Failure to adhere to established hospital patient care policy. For example, an eligible inpatient not on prophylaxis for deep vein thrombosis/pulmonary embolism whose crisis event was due to a pulmonary embolus.

- Delay in dealing with patient needs or calling for help, including an at least 20-min delay in activating a MET/RRS response, when any one or more of the objective MET activation criteria were observed.

- Inadequate monitoring (devices) and/or surveillance (personnel). For example, a patient with known symptomatic arrhythmias not on cardiac monitor or vital sign checks ordered for a patient but which are not documented in accordance with the order.

- Procedure/surgical complication coincident to cardiopulmonary arrest.

\section{Statistical analysis}

We used SPSS (version 14) to analyse the data. The $\chi^{2}$ test was used to compare proportion of predictable and avoidable arrest events, as well as outcomes across the three unit settingsunmonitored, monitored and ICUs. All p values are two-tailed and those $<0.05$ were considered significant.

\section{RESULTS}

Our MET/RRS was activated 1942 times between 1 January and 31 December 2005. Of these MET calls, 111 were cardiopulmonary arrest events (3.26 arrest events/1000 patient admissions) and 1831 were crisis events (53.8 crisis events/1000 patient admissions). The 111 arrest events occurred in 104 patients. For patients who had more than one arrest $(n=7)$, only the first cardiac arrest event was considered for analysis. Table 1 shows the patient and arrest event characteristics. There were 22 arrests (1.97 arrests/1000 patient admissions) in the unmonitored settings, 52 in monitored settings (3.38 arrests/ 1000 patient admissions), and 30 in ICU settings (4.02 arrests/ 1000 patient admissions) (table 1 ). There was a trend toward increasing "predictability" and decreasing "potential avoidability" as the level of patient monitoring increased, but in both cases it was not significant (table 2).

Overall, 26 patients survived to discharge (table 1). Table 3 shows the immediate and short-term survival after cardiopulmonary arrest events in each of the three different unit settings. The rate of immediate survival after the arrest decreased as the level of patient monitoring decreased from ICUs to monitored units to unmonitored units $(83 \%, 69 \%$ and $36.4 \%$, respectively; $\chi^{2}=12.93, p=0.002$ ) but the rate of subsequent death in hospital was higher in the more intensely monitored settings $\left(60 \%, 38 \%\right.$ and $23 \%$ respectively; $\left.\chi^{2}=7.62, p=0.022\right)($ table 3$)$. Of the 18 ICU patients who survived the arrest, the $11(61 \%)$ who died while still in hospital did so within $24 \mathrm{~h}$ of the event, whereas only 5/20 (25\%) in the monitored units and 2/5 (40\%) in unmonitored units died within $24 \mathrm{~h}$ of the event. Survival to discharge in the three settings did not differ significantly $(23 \%$, $31 \%$ and $13.6 \%$, respectively; $\chi^{2}=2.48, p=0.289$ ) (table 3 ). Of 26 patients who survived to discharge, 24 (92\%) had good neurological outcome (Cerebral Performance Category 1 or 2, or no change from baseline neurological status). Fourteen (54\%) patients were transferred to a subacute rehabilitation facility or a nursing home, eight $(31 \%)$ patients were transferred to a 
Table 1 Characteristics of patients and the cardiopulmonary arrest events $(n=104)$ included in the study

\begin{tabular}{ll}
\hline Variable & Observed value \\
\hline Age (years), mean (SD), range & $64.76(17.2), 18-93$ \\
Gender, $\mathrm{n}(\%)$ & $56(54)$ \\
Male & $48(46)$ \\
Female & \\
First observed rhythm, n (\%) & $24(23)$ \\
Ventricular tachycardia/ventricular fibrillation & $14(13.5)$ \\
Pulseless electrical activity & $42(40.5)$ \\
Asystole & $24(23)$ \\
Unknown & $22(21)$ \\
Setting, $\mathrm{n}(\%)$ & $52(50)$ \\
Unmonitored & $30(29)$ \\
Monitored & $25(24)$ \\
Intensive care & $17(16.3)$ \\
Primary service, n (\%) & $13(12.5)$ \\
General internal medicine & $13(12.5)$ \\
Cardiology & $6(5.8)$ \\
Cardiothoracic surgery & $5(4.8)$ \\
Neurosurgery & $14(13.5)$ \\
General surgery & $11(10.6)$ \\
Intensive care medicine & $35(33.7)$ \\
Other medical service* & $18(17.3)$ \\
Other surgical service $\dagger$ & $23(22.1)$ \\
Outcome, $\mathrm{n}$ (\%) & $2(1.9)$ \\
Died during event & $26(25)$ \\
Survived event, died within $24 \mathrm{~h}$ & \\
Survived event, died within 30 days & \\
Survived event, died after 30 days & \\
Survived to discharge & \\
\hline
\end{tabular}

*Geriatric medicine, pulmonary medicine, neurology, gastroenterology, haematology-oncology, physical medicine and rehabilitation. †Trauma surgery, otolaryngology, gastrointestinal surgery, transplant surgery.

long-term acute care facility, and four (15\%) patients went home (all of whom had arrested on a monitored unit).

Nineteen (18\%) cardiopulmonary arrests were considered to be "potentially avoidable". The three most common reasons for avoidability were: failure to implement established hospital guideline or policy; inadequate patient monitoring and/or surveillance; and delays in dealing with patient needs or initiating MET call (table 4). However, there were several idiosyncratic characteristics of each of these events and we have therefore listed patient and event characteristics for each of the potentially avoidable cardiopulmonary arrests in table 4 .

In all, $21(20 \%)$ patients had an arrest within $24 \mathrm{~h}$ of hospital admission; $12(57 \%)$ of these arrests were in an ICU, $8(38 \%)$ were in a monitored setting, and 1 (5\%) patient had no continuous monitoring. In none of cases was failure to place a do not resuscitate (DNR) and /or do not intubate (DNI) order the reason for the cardiopulmonary arrest call being made.

\section{DISCUSSION}

Our overall event rate of cardiopulmonary arrests (3.26 arrests/ 1000 patient admissions) is lower than those reported from other academic tertiary care hospitals, ${ }^{14}{ }^{18}$ and lower than our previously reported rate of 5.4 arrests/1000 admissions. ${ }^{16}$ The decline in the rate of arrests is due, in part, to improvements in the RRS at our institution: improved knowledge of the objective criteria for MET/RRS activation; better adherence to policy to call the team; and fewer delays to treatment in crisis situations. ${ }^{12} 21$ We have attempted to detect critical physiological deterioration in every patient before a cardiopulmonary arrest occurs, and to bring a team of responders headed by a CCM faculty member to the bedside to match resources to patient needs. The present review was designed to identify
Table 2 Cardiopulmonary arrest event "predictability" and "potential avoidability" as a function of intensity of patient monitoring. Values are number ( $\%$ of location total)

\begin{tabular}{llllll}
\hline & \multicolumn{4}{l}{ Location of arrest } \\
\cline { 2 - 6 } & $\begin{array}{l}\text { Unmonitored } \\
\text { units }\end{array}$ & $\begin{array}{l}\text { Monitored } \\
\text { units }\end{array}$ & ICUs & $\chi^{2}$, p value \\
\hline $\begin{array}{l}\text { Predictable arrest events } \\
\begin{array}{l}\text { Potentially avoidable } \\
\text { arrest events }\end{array}\end{array}$ & $6(27)$ & $17(33)$ & $14(47)$ & $4.65,0.098$ \\
\hline
\end{tabular}

*Test for comparison of proportion of predictable and potentially avoidable arrests across the three unit settings.

potentially avoidable arrests and study the reasons for avoidability. This has not been studied before in a MET/RRS setting.

Our data show that our goal of eliminating all avoidable cardiopulmonary arrests has not been reached. Nearly half of all arrests occurred in a monitored setting (table 1). The "predictability" of arrests seemed to increase, and "potential avoidability" tended to decrease as the level of patient monitoring increased (from unmonitored units to monitored units to ICUs), although this trend was not significant with the small sample size (table 2). More patients who had an arrest on monitored and intensive care units survived the initial event (table 3). One could expect this because monitoring devices (including continuous pulse oximetry and telemetry devices) allow early detection of patient deterioration and alert caregivers to the need for an immediate clinical response. The higher rate of resuscitation in monitored settings, however, did not lead to a higher survival to discharge, which was similar across all three unit settings (table 3 ). In the ICU, most patients $(61 \%)$ who survived the event died within the next $24 \mathrm{~h}$, but in monitored units $25 \%$ died within the next $24 \mathrm{~h}$. Many of the patients on monitored units who survived the initial 24-h period after an arrest succumbed later while still in hospital.

Arrests on unmonitored units presented a different story. Nearly two-thirds of these patients did not survive the event (table 3). The most likely explanation seems to be that detection of the cardiopulmonary arrest state may be delayed in patients who are not continuously monitored. A majority of unmonitored patients had sustained an unwitnessed cardiopulmonary arrest and the unexpected collapse was discovered by medical personnel during a routine visit to the patient room. In particular, four patients (cases 14, 15, 17, 18; table 4) of six (cases 14-19) whose arrest on an unmonitored unit was deemed "potentially avoidable", might have benefited from a more intense monitoring environment (table 4). However, conclusions based on small numbers are, of course, speculative.

We found that 16.5 crisis MET calls were made for each cardiopulmonary arrest call (53.8 MET "crisis" calls/1000

Table 3 Outcome after cardiopulmonary arrest as a function of intensity of patient monitoring.

\begin{tabular}{|c|c|c|c|c|}
\hline & \multicolumn{4}{|c|}{ Location of arrest } \\
\hline & $\begin{array}{l}\text { Unmonitored } \\
\text { units }\end{array}$ & $\begin{array}{l}\text { Monitored } \\
\text { units }\end{array}$ & ICUs & $\chi^{2}, \mathrm{p}$ value $^{*}$ \\
\hline \multirow{4}{*}{$\begin{array}{l}\text { Died during event } \\
\text { Survived event, } \\
\text { died in hospital } \\
\text { Survived to } \\
\text { discharge } \\
\text { Total }\end{array}$} & $14(63.6)$ & $16(31)$ & $5(17)$ & $12.93,0.002$ \\
\hline & $5(22.7)$ & $20(38)$ & $18(60)$ & $7.62,0.022$ \\
\hline & $3(13.6)$ & $16(31)$ & $7(23)$ & $2.48,0.289$ \\
\hline & $22(100)$ & $52(100)$ & $30(100)$ & \\
\hline
\end{tabular}




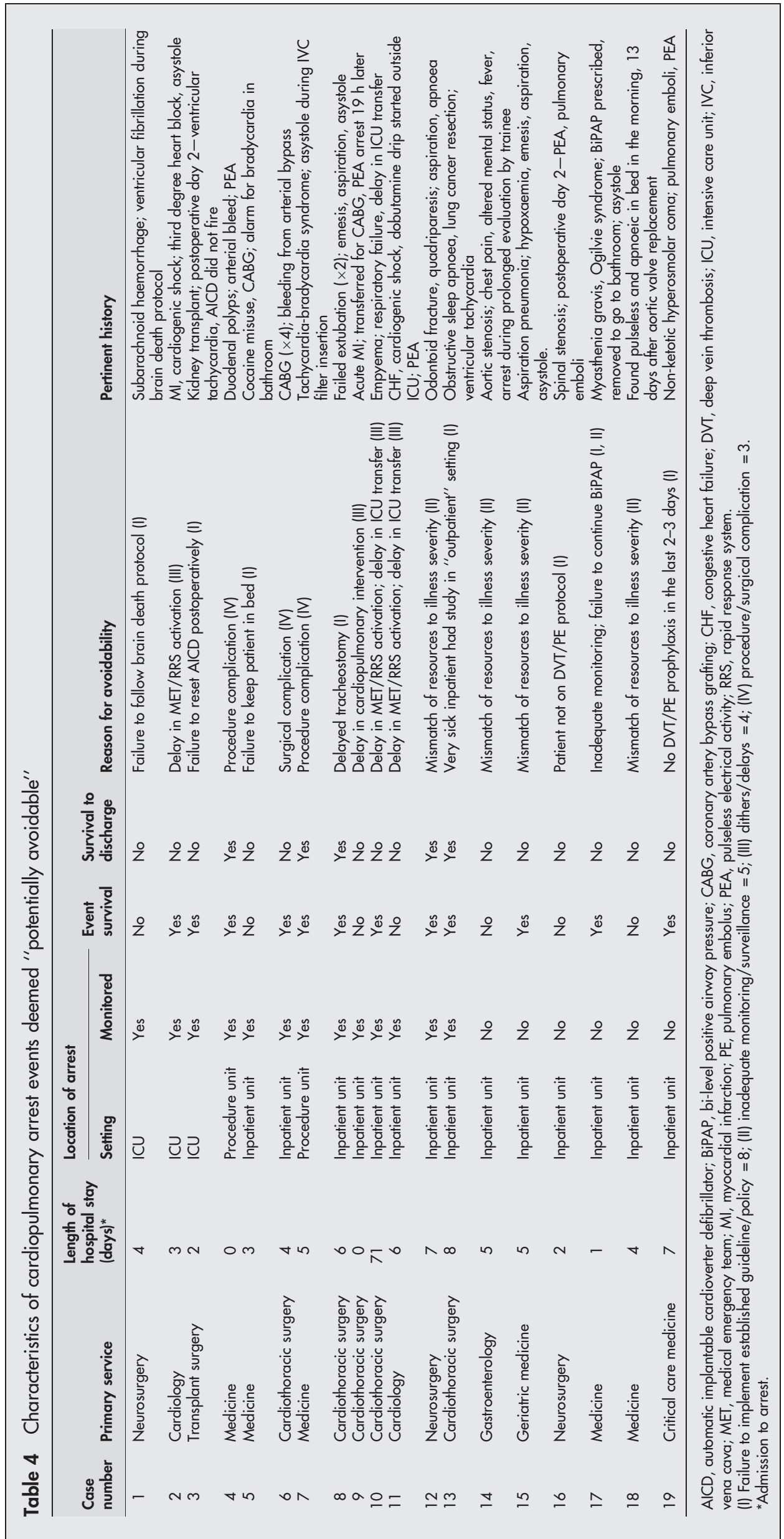


patient admissions vs 3.26 MET "arrest" calls/1000 patient admissions). Despite the high crisis event to arrest ratio, we still found that nearly 1 in 5 arrests was potentially avoidable. Of the five patients who had potentially avoidable arrest and survived to discharge, two patients (cases 4, 7; table 4) had cardiopulmonary complications of procedures in specialised interventional suites-areas in which physiological monitoring and critical care resources can be inadequate; two patients (cases 8,12 ) had potentially avoidable respiratory obstructions; and one patient (case 13) was transferred for testing after pulmonary resection to an inhospital outpatient sleep laboratory. In these five cases, the MET response was life saving. None of the other 14 patients with "potentially avoidable" arrests survived to discharge. Six patients (cases 14-19; 43\%) were in unmonitored beds yet four patients (cases 14, 15, 17, 18) had medical indications for being in a monitored setting; care providers in two others(cases 16, 19) deviated from accepted practice for prophylactic anticoagulation for deep vein thrombosis/pulmonary embolus. Eight of the patients who did not survive their arrest (cases 1, 2, 3, 5, 6, 9-11) were in monitored and intensive care units. One of these (case 1) was in the process of death determination using neurological criteria and had a cardiopulmonary arrest during apnoea testing. Three patients (cases 2, 10, 11) were in monitored settings and met our criteria for initiating a MET response, but there was delay in activating a MET response ranging from $20 \mathrm{~min}$ to an hour. Three others (cases 3, 5, 9) had a deviation from a well-defined plan of treatment prior to the arrest: one patient (case 3) with an automatic implantable cardioverter defibrillator (AICD) in place did not have the AICD reset after an operation and had ventricular tachycardia arrest; another patient (case 5) with history of drug misuse was found locked in the bathroom in asystolic arrest; a third patient (case 9) had a myocardial infarction and had a pulseless electrical activity (PEA) arrest while waiting to go to the cardiac catheterisation laboratory. The last patient in this group (case 6) had bleeding from a recently placed coronary artery bypass graft, arrested in the ICU, and had to be taken back to the operating room for surgical repair.

Thus, the most common circumstances of avoidable arrests that we found were sudden critical illness in under-monitored patients, delays in initiating MET response for monitored patients who met crisis criteria, and failure to follow best practices in respiratory care or thromboembolism prophylaxis. Delay in MET/RRS activation and failure to follow "best practices" can be dealt with by a rigorous programme of continuing education for all staff. We have implemented a programme called "The first five minutes" (F Tasota, personal communication, 2007). This programme uses simulated patient crisis scenarios to teach nursing staff how to recognise a crisis, how to trigger the RRS and what to do while waiting for the MET responders. We have and continue to provide simulationbased training to all internal medicine, critical care, pulmonary medicine, psychiatry and cardiology trainees on how to trigger and participate in a patient crisis response. ${ }^{24}$ The personnel and device aspects of monitoring were not analysed separately as part of this project, although we believe that doing so would be a meritorious undertaking in another study. For example, a focused audit of the impact of the quality and frequency of nurse recording of patient vital sign information on crisis detection would be interesting. The issue of whether expanded patient monitoring using automated devices would further reduce the incidence or consequences of true cardiopulmonary arrests could also be separately studied. Unmonitored settings are inadequately equipped to detect physiological deterioration, but costs and potential benefits of universal inpatient monitoring using automated devices have not been adequately studied.
The improvement in immediate and short-term survival after cardiopulmonary arrest in the present study supports the idea that proper monitoring of high-risk patients in monitored and intensive care units might prevent immediate adverse outcomes.

Of 21 patients who had an arrest within $24 \mathrm{~h}$ of hospital admission, 20 were in an appropriately monitored setting with adequate resources and expertise at hand; the one patient who arrested in an unmonitored setting had an arrest that was deemed "unpredictable and unavoidable". Thus, inappropriate triage for new hospital admissions did not seem to be a problem in this group of 104 patients. Failure to discuss end-of-life care in a timely fashion has been suggested as a cause of potentially avoidable cardiac arrests (D Jones, personal communication, 2006). However, this was not true for cases in our review. Local culture will affect the types and frequency of potentially avoidable cardiac arrests, and whereas some hospitals may benefit from an initiative aimed at improving end-of-life care, at our hospital and may be at some others, the benefit is expected to be less.

\section{Limitations of the study}

The present study was a quality improvement project that included a careful but retrospective chart review. Some pertinent data may not have been recorded or were not available in patient charts although this is unlikely. We identified cardiac arrest events based on the caregivers' call for a cardiopulmonary arrest (condition A). It is possible that some arrest events were misclassified as crisis (condition C) events and not included in our analyses. However, in our sampling of over 1000 crisis events during the same calendar year, we found that only five arrest events were misclassified as non-arrest crisis events. These five events have been included in our analysis. It is also possible that at times when an inpatient has a cardiopulmonary arrest the MET/RRS is not activated because all the required expertise is already available at bedside. This is more likely in the ICU; we have not previously discovered a cardiac arrest outside the ICU that did not have either a condition C or A call. Therefore, we probably did not "miss" any cardiopulmonary arrests due to this factor. Lastly, we acknowledge that the subjective judgment of experts was used to categorise events as "predictable" and "potentially avoidable", and to determine reasons for avoidability. We report these findings because they may stimulate discussion regarding the limits of MET/RRS to eliminate avoidable inhospital cardiopulmonary arrest events, and the need to use other methods to accomplish the goal of avoiding all preventable inhospital cardiopulmonary arrests.

\section{CONCLUSIONS}

Our "mature" RRS did not eliminate all the potentially avoidable causes of inpatient cardiopulmonary arrests. We found evidence to support the hypothesis that more frequent inpatient monitoring, and improved caregiver adherence to established guidelines (including timely MET/RRS activation following patient deterioration), might either prevent or improve the outcome of some cardiopulmonary arrests. Additional MET/RRS calls might not prevent more cardiopulmonary arrests.

\section{ACKNOWLEDGEMENTS}

We would like to thank Drs Jason Leong and Stephanie Freeman for their support in data collection.

\section{MEMBERS OF THE MERIT COMMITTEE}

Joanne Woodson; Lori L Bashline; Alan Brytus; Carol Mcbroom; Christine Wu; Colleen Carroll; Daniel Shearn; Joseph Darby; Darla Annonio; Rosemary Debar; David P Deen; Edgar Delgado; Jeffrey J 
Alvarez Jr; Mark J Barnhouse; Angela Bayless; David Bertoty; Paulette Bingham; Laurie Buches; Leslie Cairns; Michelle R Ciccone-Clayton; Marsha A Daychak; Carol Elsesser; Elaine Farmer; Laura A Fennimore; Kim Fleegle; Mary M Foster-Heasley; Lisa Fox-Hawranko; Susan Frank; Tracy Grogan; Kathy Guentner; Linda Haas; Cathy Hammel; Katherine Hannan; Mary Hrinya; Marilyn Hudak; Jill Huwe; Heidi Karalagas; Diane Kielur; Caroline Kissell; Mary Kissler; Diane Kulas; Cassandra Lawrence; Deborah Mangol; Valerie Mastren; Marianne McConnell; Roxanne Mcwreath; David Miller; Jeff Murray; Michelle Nagy; Eary Ott; Christina L Paganelli; Mary Beth Pais; Anita Payne; Kymberli Potersnak; Laurie Rack; Debra Santarelli; Carol Scholle; Daniel Shearn; Susan Simms; Joann Slade; Melanie Smith; Nancy Smith; Lori Snyder; Mary Staller; Cynthia Valenta; Donna Ward; Sue Wesmiller; Janice Zagari; Tom Dongilli; Melinda Fiedor; Karen Fielding; Patricia Geraci; Karen Gilchrist; Camellia Herisko; Nancy Hudacko; Norma M Jackson; Janice Phillipps; Joanne Kowiatek; Marlon Johnson; Judi Hanna; Keith Radakovich; Mary Kennedy; John Kreit; Wolff, Kristine Keefer; Jason R Leong; Linda Haas; Anthony Liscotti; Lydia Mansur; Marilyn Hudak; David McAdams; Karan Moore; Holt Murray; Nicholas Bircher; Donna Ottoviani; Paul Beam; Paula Natale; Andrea Schmid; Carol Scholle; Joann Slade; Richard Snyder; Melissa Sroka; Catherine Stepien; Sue Svec; Frederick J Tasota; Thomas Smitherman; Cindy Valko; Misana Vantosky; Vincent Mosesso Jr; Catherine Vitari; Wendeline Grbach.

\section{Authors' affiliations}

Sanjay Galhotra, Michael A DeVita, Mary Amanda Dew, University of Pittsburgh, Pittsburgh, Pennsylvania, USA

Richard L Simmons, Members of the Medical Emergency Response Improvement Team (MERIT) CommitteeUniversity of Pittsburgh Medical Center Presbyterian Hospital, Pittsburgh, Pennsylvania, USA

Competing interests: None.

\section{REFERENCES}

1 Lazzam C, McCans JL. Predictors of survival of in-hospital cardiac arrest. Can J Cardiol 1991;7:113-6.

2 Herlitz J, Bang A, Aune S, et al. Characteristics and outcome among patients suffering in-hospital cardiac arrest in monitored and non-monitored areas. Resuscitation 2001;48:125-35.

3 Dumot JA, Burval DJ, Sprung J. Outcome of adult cardiopulmonary resuscitations at a tertiary referral center including results of "limited" resuscitations. Arch Intern Med 2001;161:1751-8.

4 Schneider AP II, Nelson DJ, Brown DD. In-hospital cardiopulmonary resuscitation: a 30-year review. J Am Board Fam Pract 1993;6:91-101.
5 Peberdy MA, Kaye W, Ornato JP, et al. Cardiopulmonary resuscitation of adults in the hospital: A report of 14,720 cardiac arrests from the National Registry of Cardiopulmonary Resuscitation. Resuscitation 2003;58:297-308.

6 Nadkarni VM, Larkin GL, Peberdy MA, et al. First documented rhythm and clinical outcome from in-hospital cardiac arrest among children and adults. JAMA 2006;295:50-7.

7 Association of American Medical Colleges. Institute for Improving Clinical Care.IICC Initiatives. Academic Rapid Response Team Collaborative. http:// www.aamc.org/patientcare/iicc/initiatives.htm (accessed 6 December 2006).

8 Berwick DM, Calkins DR, McCannon CJ, et al. The 100,000 Lives Campaign: setting a goal and a deadline for improving health care quality. JAMA 2006;295:324-7.

9 DeVita MA, Bellomo R, Hillman K, et al. Findings of the First Consensus Conference on Medical Emergency Teams. Crit Care Med 2006;34:2463-78.

10 Schein RMH, Hazday N, Pena M, et al. Clinical antecedents to in-hospital cardiopulmonary arrest. Chest 1990;98:1388-92.

11 Smith AF, Wood J. Can some in-hospital cardio-respiratory arrests be prevented? A prospective survey. Resuscitation 1998;37:133-7.

12 Hodgetts TJ, Kenward G, Vlackonikolis L, et al. Incidence, location and reasons for avoidable in-hospital cardiac arrest in a district general hospital. Resuscitation 2002;54:115-23.

13 Nurmi J, Harjola VP, Nolan J, et al. Observations and warning signs prior to cardiac arrest. Should a medical emergency team intervene earlier? Acta Anaesthesiol Scand 2005;49:702-6.

14 Buist MD, Moore GE, Bernard SA, et al. Effects of a medical emergency team on reduction of incidence of and mortality from unexpected cardiopulmonary arrests in hospital: a preliminary study. BMJ 2002;324:387-90.

15 Bellomo R, Goldsmith D, Uchino S, et al. A prospective before-and-after trial of a medical emergency team. Med J Aust 2003;179:283-8.

16 DeVita MA, Braithwaite RS, Mahidhara R, et al. Use of medical emergency team responses to reduce hospital cardiopulmonary arrests. Qual Saf Health Care 2004; 13:251-4.

17 Hillman K, Chen J, Cretikos M, et al. Introduction of the medical emergency team (MET) system: a cluster-randomised controlled trial. Lancet 2005:365:2091-7.

18 Jones D, Bellomo R, Bates S, et al. Long term effect of a medical emergency team on cardiac arrests in a teaching hospital. Crit Care 2005;9:R808-15.

19 Jones D, Bellomo R, Bates S, et al. Patient monitoring and timing of cardiac arrests and medical emergency team calls in a teaching hospital. Intensive Care Med 2006;32:1352-6.

20 Galhotra S, DeVita MA, Simmons RL, et al. Impact of patient monitoring on the diurnal pattern of Medical Emergency Team activation. Crit Care Med 2006;34:1700-6.

21 Foraida MI, DeVita MA, Braithwaite RS, et al. Improving the utilization of medical crisis teams (Condition C) at an urban tertiary care hospital. J Crit Care 2003; 18:87-94.

22 Braithwaite RS, DeVita MA, Mahidhara R, et al. Use of medical emergency team (MET) responses to detect medical errors. Qual Saf Health Care 2004;13:255-9.

23 Jennett B, Bond M. Assessment of outcome after severe brain damage: a practical scale. Lancet 1975; i:480-4.

24 DeVita MA, Schaefer J, Lutz J, et al. Improving medical emergency team (MET) performance using a novel curriculum and a computerized human patient simulator. Qual Saf Health Care 2005;14:326-31.

\section{Access a vast information database with Toll-Free linking}

"Toll-free" linking gives you immediate access to the full text of many of the cited articles in a paper's reference list-FOR FREE. With the support of HighWire's vast journal catalogue, a huge reference library is now open to you. If HighWire hosts the journal, you can view the full text of the referenced article, completely free of charge by following the Free Full Text links in the references. 